\title{
Special Issue on Three-Dimensional Structural Modeling
}

\author{
Guillaume Caumon • Pauline Collon-Drouaillet
}

Published online: 30 October 2014

(C) International Association for Mathematical Geosciences 2014

Three-dimensional (3D) structural models are generalizations of geological maps and cross-sections used by geologists for the last 200 years. They describe the geometry and layout of rock units in 3D space by honoring available observations, measurements and geological concepts. They are used on a daily basis in industry and academia to improve one's understanding of an area, to quantify geological processes and to address natural resource management problems (oil and gas, mining, geothermal energy, ground water). This area of research raises interesting questions concerning the ways to build these models and to appropriately describe, analyze and reduce the associated uncertainty. For these ends, new conceptual models relating to geological processes and geometric parameters are needed. This issue gathers contributions regarding all of these aspects.

In preparing this special issue, we have received a total of 25 paper proposals and 13 full papers, of which 5 are published in the present issue and 2 are still in revision. We would like to thank all the authors for their contributions and the reviewers for both their help and remarks, which have been essential to the editorial process.

3D structural modeling emerged in the 1970s by generating computerized depth maps with contouring techniques. These methods are very effective and are still in use today for the processing and analysis of topographic surfaces and depth maps. However, they raise problems when it comes to fault management, or when dealing with complex surfaces having several Z-values for the same geographic coordinate (e.g., recumbent folds, salt tops). This limitation motivated the development of techniques

\footnotetext{
G. Caumon ( $\square) \cdot$ P. Collon-Drouaillet

GeoRessources UMR 7359 Université de Lorraine-CNRS-CREGU, ENSG,

2 rue du Doyen Marcel Roubault, 54518 Vandoeuvre-lès-Nancy cedex, France

e-mail: Guillaume.Caumon@univ-lorraine.fr

P. Collon-Drouaillet

e-mail: Pauline.Collon@univ-lorraine.fr
} 
similar to computer-aided design during the 1990s. These methods operate on more general descriptions of geological surfaces: parametric surfaces (De Kemp 1999) and triangulated surfaces (see Caumon et al. 2009 and references therein). They combine automatic and interactive approaches to connect data and interpretations represented as lines and points in space, and allow for the editing of 3D surfaces under constraints. They have a very good ability to represent all sorts of complex geological structures, but often require a significant amount of user interaction. More recently, volumetric approaches have been proposed to foster more automation and stability in 3D modeling tasks (e.g. Calcagno et al. 2008; Frank et al. 2007; Hjelle and Petersen 2011; Souche et al. 2013).

This special issue presents two contributions in 3D structural model building. Chuanfa Chen, Yanyan Li, Xuewei Cao and Honglei Dai focus on parametric surface modeling and smoothing problems in the context of noisy elevation data. They propose a method based on least-squares thin plate splines, which uses regularization to ensure satisfactory numerical behavior of the minimization (avoiding over-fitting while ensuring the system is well conditioned). This new method is particularly adapted to DEM construction from noisy data and is demonstrated on two real case studies from China.

Michael Hillier, Ernst Schetselaar, Eric de Kemp and Gervais Perron revisit volumetric structural modeling by using a general radial basis function formalism. As field data often gives little direct information about stratigraphic contacts, they propose to incorporate gradient, inequality and axial measurement constraints by casting the implicit surface problem in this general framework. Thanks to an original computation of orientation matrices, they also locally compute anisotropy parameters over one or multiple domains. The efficiency of such new tools in sparse data environments is demonstrated through two synthetic examples, showing a promising ability to automate 3D structural modeling of complex folds in sparse data settings.

Whatever the modeling method, the level of interpretation (as opposed to observations) significantly increases between maps, cross-sections and 3D structural models. In classical geology, the methods to build cross-sections by hand are taught in all undergraduate classes and are well accepted. 3D modeling has not reached a comparable stage yet. Indeed, 3D modeling still raises suspicion from some geologists who remain skeptical about mathematics and numerical computations and instead prefer sound conceptual thinking and well-accepted methodologies implemented with paper and pencil. As a matter of fact, 3D numerical approaches do not replace geological thinking, but should help reflection, especially in non-cylindrical settings and in domains that are highly compartmentalized by faults. Nonetheless, a typical (and valid) question directed towards authors of 3D modeling case studies is: "What is the uncertainty relative to this model?". It is generally impossible to answer this question rigorously on a hand-made cross-section, but probability theory can be used on $3 \mathrm{D}$ models to generate alternative structures. A second related question is "How can I reduce uncertainty?". Two papers featured in this issue of Mathematical Geosciences provide interesting elements to answer these challenging questions.

Per Røe, Frode Georgsen and Petter Abrahamsen propose a model to describe fault uncertainty based on tilted grids, p-field simulation and Gaussian random fields. Their approach simulates alternative geometries within a fault envelope (which can be 
inferred from seismic data), while a variogram allows for controlling the smoothness of the perturbation. In particular, not only do they propose ways to efficiently honor several types of well observations when generating several stochastic fault surfaces, but they also demonstrate the overall methodology on a complex reservoir data set.

Mark Lindsay, Stéphane Perrouty, Mark Lessell and Laurent Aillères propose a new workflow to provide geologically consistent models that cover the uncertainty space as an input for geophysical inversion. Upon defining metrics to characterize the geodiversity, they use Principal Component Analysis to identify representative models of the geological diversity that can explain geophysical potential field data. In doing so, they are able to produce multiple geological realizations that constitute a new input set for classical potential field inversion, which are based on perturbations of depth map or petrophysical parameters. Their application on a real dataset in West Africa demonstrates how the proposed approach improves both the inversion process and the uncertainty assessment.

Nonetheless, the question regarding the ability of mathematical methods to appropriately describe the inference process used in geological interpretation tasks remains. A possible way of addressing this problem is to better connect observed structural features with deformation processes. This is exactly what Timothy Dodwell and Giles Hunt propose in the last paper of this issue. Beginning with field observation of chevron folds, they propose a mathematical formulation to explain periodic structures occurring in folds, which is based on geomechanical principles. This extended formulation includes boundary conditions at points of layer separation and integrates effects of axial loads. This results in the definition of an energy-based model that reproduces the periodic voids appearing in such a particularly constrained context. The formation of such geometrical features thus appears as the result of a subtle interplay of geometric constraints (layers forced to fit together) and the mechanical properties of the layers themselves.

When compared to professionals belonging to other fields of mathematical geosciences, such as geostatistics, most researchers who focus on 3D geological modeling methods operate in an industry in which journal publication is not typically a priority. This means that a significant part of research results in this field are only accessible from succinct conference papers, patents and software manuals. We hope that the papers on 3D structural modeling presented in this issue of Mathematical Geosciences will help researchers and practitioners in their work and will contribute to the dissemination of ideas in this exciting field.

\section{References}

Calcagno P, Chilès JP, Courrioux G, Guillen A (2008) Geological modelling from field data and geological knowledge: Part I. Modelling method coupling 3D potential-field interpolation and geological rules. Phys Earth Planet Inter 171(1):147-157

Caumon G, Collon-Drouaillet P, de Veslud CLC, Viseur S, Sausse J (2009) Surface-based 3D modeling of geological structures. Math Geosci 41(8):927-945

De Kemp EA (1999) Visualization of complex geological structures using 3-D Bezier construction tools. Comput Geosci 25(5):581-597

Frank T, Tertois AL, Mallet JL (2007) 3D-reconstruction of complex geological interfaces from irregularly distributed and noisy point data. Comput Geosci 33(7):932-943 
Hjelle $\varnothing$, Petersen SA (2011) A Hamilton-Jacobi framework for modeling folds in structural geology. Math Geosci 43(7):741-761

Souche L, Lepage F, Iskenova G (2013) Volume based modeling-automated construction of complex structural models. In: 75th EAGE conference and exhibition incorporating SPE EUROPEC. doi:10.3997/ 2214-4609.20130037 